\title{
MicroRNA-429 inhibits cancer cell proliferation and migration by targeting AKT1 in renal cell carcinoma
}

\author{
ZHENGMING SU $^{1,2^{*}}$, GANGGANG JIANG $^{1,3^{*}}$, JINLAN CHEN $^{1}$, XING LIU $^{1}$, HAIBO ZHAO $^{1}$, \\ ZHIYUAN FANG ${ }^{3}$, YONGZHONG HE ${ }^{1}$, XIANHAN JIANG ${ }^{1,2}$ and GUIBIN XU ${ }^{1,2}$ \\ ${ }^{1}$ Department of Urology, The Fifth Affiliated Hospital of Guangzhou Medical University; ${ }^{2}$ Center for the \\ Innovation and Translation of Minimally Invasive Techniques, Guangzhou Medical University; \\ ${ }^{3}$ Department of Center Laboratory, The Fifth Affiliated Hospital of Guangzhou \\ Medical University, Guangzhou, Guangdong 510700, P.R. China
}

Received March 24, 2019; Accepted September 19, 2019

DOI: $10.3892 /$ mco.2019.1940

\begin{abstract}
MicroRNAs (miRNAs or miR) serve as oncogenes and tumor suppressors. In a previous study, it was revealed that has-miRNA-429 (miR-429) is a tumor suppressor in 786-O renal cell carcinoma (RCC) cells. However, its mechanism in $\mathrm{RCC}$ remains to be determined. The present study aimed to explain the functional role and mechanism of miR-429 in RCC pathogenesis. Luciferase reporter assays demonstrated that miR-429 overexpression reduced the transcriptional activity of AKT serine/threonine kinase 1 (AKT1). Reverse transcripton-quantitative (RT-q) PCR and western blot analysis indicated that the mRNA and protein expression of AKT1 was downregulated in 786-O RCC cell lines when miR-429 was overexpressed, indicating that miR-429 may directly target AKT1 in RCC. Therefore, miR-429 overexpression enhanced the inhibition of tumor size and weight in nude mice in vivo. The current study indicated that the novel miR-429-regulated pathway may provide insights into RCC oncogenesis and metastasis.
\end{abstract}

\section{Introduction}

Renal cell carcinoma (RCC) accounts for $2-3 \%$ of all adult malignancies, and its incidence has an increasing rate of $2-3 \%$ per decade (1). Approximately $80 \%$ of patients with RCC worldwide are diagnosed with clear cell RCC (ccRCC),

Correspondence to: Professor Xianhan Jiang or Professor Guibin Xu, Department of Urology, The Fifth Affiliated Hospital of Guangzhou Medical University, 621 Gangwan Road, Guangzhou, Guangdong 510700, P.R. China

E-mail: jiangxianhangz@126.com

E-mail: gyxgb@163.com

*Contributed equally

Key words: renal cell carcinoma, AKT serine/threonine kinase 1, microRNA-429 the most common subtype of RCC. Although surgery is still the major modality for RCC, $50 \%$ of patients with RCC still suffer from recurrence with distant metastasis after curative nephrectomy (2). Biomarkers for the early diagnosis and targeted therapies of RCC are currently lacking. Therefore, precise, sensitive biomarkers and the targeted treatment for RCC therapy are urgently needed.

MicroRNAs (miRNAs) are small endogenous ncRNA comprising 19-22 nucleotides that regulate gene and protein-coding gene expression levels at the post-transcriptional level through translational inhibition or mRNA degradation (3). miRNAs are distributed in some cancer types (4-6) and dysregulate the key processes of tumor development and progression, including proliferation, differentiation, cell cycle, apoptosis, migration, and invasion $(7,8)$.

miR-429 is a member of the miR-200 family and an oncogene or tumor-suppressor gene in diverse cancer types, including ovarian cancer (9), cervical cancer (10), cholangiocarcinoma (11), non-small cell lung cancer (12), gastric cancer (13), and hepatocellular carcinoma (14). Wu et al (15) found that the miR-429 in RCC contributes to cell migration, survival, and invasion via the downregulation of Sp1. Chen et al (16) also reported that miR-429 can regulate RCC cell function by targeting VEGF and reduce the 3'-untranslated region (3'-UTR) luciferase action of VEGF. However, the role and mechanisms of miR-429 in HCC have not been elucidated. In this study, we identified AKT1 as a target of miR-429 and showed the possible role of miR-429/AKT1-mediated signal pathway as a new target for RCC treatment.

\section{Materials and methods}

Cell culture. Cells 786-O and 293T were purchased from the American Type Culture Collection (Manassas, VA, USA), cultured in DMEM (Invitrogen Life Technologies; Thermo Fisher Scientific, Inc., Waltham, MA, USA), and supplemented with $10 \%$ fetal bovine serum (Shanghai ExCell Biology, Inc., Shanghai, China), $100 \mathrm{U} / \mathrm{ml}$ penicillin, and $100 \mu \mathrm{g} / \mathrm{ml}$ streptomycin. The cultures were maintained at $37^{\circ} \mathrm{C}$ in a humidified air atmosphere with $5 \% \mathrm{CO}_{2}$. 
Cell transfection. The miR-429 mimics, miR-429 inhibitor, negative control miRNA mimics (mimics $\mathrm{NC}$ ), and negative control miRNA inhibitor (inhibitor NC) were chemically synthesized by Shanghai GenePharma Co., Ltd. (Shanghai, China). Some sequences are listed in Table I. 293T and 786-o cells of $60-80 \%$ confluence were transfected with miR-429 mimic, NC mimic, miR-429 inhibitor or inhibitor controls using Lipofectamine 2000 reagent (Invitrogen; Thermo Fisher Scientific, Inc) according to the manufacturer's instructions. The transfection efficiency and miR-429 expression changes were confirmed by fluorescence microscopy (DMIRB; Leica Microsystems GmbH, Wetzlar, Germany) and qPCR. The culture medium was refreshed $6 \mathrm{~h}$ after transfection, and the transfection efficiency was observed in the 293T and 786-o cells that were transfected with green fluorescent protein Fam-labeled miR-429 mimic-negative control (GenePharma Co., Ltd.). The cells were harvested and the total RNA was extracted for qPCR analysis $24 \mathrm{~h}$ after transfection with a miR-429 mimic or the control.

Reverse transcription-quantitative (RT- $q$ ) PCR. Total RNA was extracted from cells by using TRIzol ${ }^{\circledR}$ reagent (Invitrogen Life Technologies; Thermo Fisher Scientific, Inc.) according to the manufacturer's instructions. RT was performed to analyze the mRNA expression levels of AKT1 by using the Revert Aid First Strand cDNA synthesis kit (Thermo Fisher Scientific, Inc., Vilnius, Lithuania) according to the manufacturer's instructions. The $20 \mu \mathrm{l}$ of PCR mixture contained $10 \mu \mathrm{l}$ of $2 \mathrm{X}$ QuantiTect SYBR Green PCR Master mix (Takara Bio, Inc., Otsu, Japan), $1 \mu \mathrm{l}$ of cDNA template, $1 \mu \mathrm{l}$ of each primer, and RNase-free water. The program for polymerase chain reaction (PCR) was as follows: $95^{\circ} \mathrm{C}$ for $15 \mathrm{~min}$, followed by 45 cycles of $94^{\circ} \mathrm{C}$ for $15 \mathrm{sec}, 57^{\circ} \mathrm{C}$ for $30 \mathrm{sec}$, and $72^{\circ} \mathrm{C}$ for $30 \mathrm{sec}$. AKT1 expression levels were evaluated using the comparative threshold cycle (Ct) method (17). GAPDH was used as an internal control. The primers for AKT1 and GAPDH are listed in Table I. Each sample was detected in triplicate.

Luciferase reporter assay. Luciferase reporter assay was used to verify whether or not AKT1 is a direct target of miR-429. The 3'-UTR region of AKT1 with the potential binding sites or mutant sites of miR-429 were generated, followed by fusion into the luciferase reporter vector pGL3-promoter vector (Promega Corporation, Madison, WI, USA, named as AKT1-WT or AKT1-MUT). Amplification was conducted using the primers listed in Table I. Then, 293T cells were seeded $(10,000$ cells/well) in a $96-$ well plate and co-transfected with miR-429 mimic/miR-negative control, miR-429 inhibitor/inhibitor control and WT/MUT-AKT1-3'-UTR vector. Luciferase activity was measured using a Luciferase reporter assay kit (Promega Corporation) according to the manufacturer's guidelines at $48 \mathrm{~h}$ after transfection.

Protein extraction and western blot analysis. Western blot was performed according to the manufacturer's guidance. The cells were homogenized, and total protein was extracted using RIPA buffer and analyzed by Western blot. The proteins were then detected with antibodies. The primary antibodies included AKT1 (1:1,000; cat no. ab-81283; Abcam, Hong Kong, China) and GAPDH (1:1,000; cat no.sc-47724; Santa Cruz
Biotechnology, Inc., Santa Cruz, CA, USA). Goat anti-rabbit IgG-HRP purchased from Beyotime was used as the secondary antibody. GAPDH was used as an internal control.

In vivo tumor xenograft studies. Six BALB/c-nu mice (6 weeks, 16-18 g) were provided by Shanghai Slac Laboratory Animal Ltd. (Shanghai, China) and were allowed to adapt for 3 days before any experimental procedures. The 786-O cells were injected into the underarms of each mouse. The mice received two intratumoral injection of miR-429 miRNA agomir or agomir negative control each week until the tumor size reached approximately $5 \times 5 \mathrm{~mm}$. Tumor size was supervised every week, and tumor volume was calculated using the formula $V=\left(a^{2} \mathrm{x} b\right) / 2$, where $a$ and $b$ represents the width and length, respectively. Finally, mice were euthanized with an intraperitoneal injection of pentobarbital sodium $(200 \mathrm{mg} / \mathrm{kg})$ at the end of the experiment, then tumors were harvested and weighed. Symptoms such as pain, weight loss, loss of appetite or weakness were set as humane endpoints for the present study; however, no animal was sacrificed before the completion of the experiment as a result of displaying any of these symptoms. The animal experiment was approved by the Institutional Ethics Committee on Animal Care and Experimentation at The Guangzhou Medical University of China (approval no. 2017-020).

Statistical analysis. Statistical significance was analyzed using the t-test. Each sample was evaluated in triplicate, and the results were expressed as the means \pm standard deviation. A two-tailed $\mathrm{P}<0.05$ was considered significant. All statistical analyses were conducted using SPSS 17.0 statistical software (SPSS, Inc., Chicago, IL, USA).

\section{Results}

miR-429 downregulated AKT1 expression in 786-O RCC cell lines. mRNA and protein analyses were performed to confirm the effect of miR-429 on AKT1 expression at the transcriptional or translational levels. Compared with the control group, the cells transfected with the miR-429 mimics suppressed the mRNA expression of AKT1 (Fig. 1A), and this finding was consistent with those of luciferase report assay. miR-429 overexpression remarkably decreased the AKT1 protein expression in 786-O RCC cell lines (Fig. 1B). The Fam-labeled negative control was transfected into the cells, and the transfection efficiency was analyzed by fluorescence microscopy $6 \mathrm{~h}$ after transfection. The transfection efficiency was $\sim 80$ and $85 \%$ in $293 \mathrm{~T}$ and $786-\mathrm{o}$ cells, respectively. In addition, the fold changes to the expression levels of miR-429, as determined by qPCR, were 130.4 and 162.3 in the 293T and 786-o cells Compared with the NC mimic control group, respectively (Fig. 1C).

$A K T 1$ is a direct target of miR-429. miRNA target was predicted using computational algorithms according to the base-pairing rules between miRNA and mRNA target sites, the location of binding sequences within the target $3^{\prime}-\mathrm{UTR}$, and the conservation of target binding sequences within associated genomes. The gene was predicted using the microRNA (http://www.microrna.org/microrna/home.do) computational algorithms (Fig. 2A). 
Table I. Primer sequences used in the present study.

\begin{tabular}{ll}
\hline Primer & \multicolumn{1}{c}{ Sequence } \\
\hline AKT1 & F: AGCGACGTGGCTATTGTGAAG \\
& R: GCCATCATTCTTGAGGAGGAAGT \\
AKT1-3'UTR-WT & F: ACGCGTGCTAGCCCGGGCGAGCCCAGATGGAAAGACGT \\
& R: GCAGATCGCAGATCTCGAATTTGGAGGGAAGGTTCCA \\
AKT1-3'UTR-MUT & F: TTTACAACATTCAACTTCGAAGAGCTTACTATTATAATATAATA \\
& R: TATTATATTATAATAGTAAGCTTCACGAAGTTGAATGTTGAAA \\
miR-429 mimic & F: UAAUACUGUCUGGUAAAACCGU \\
NC mimic & R: GGUUUUACCAGACAGUAUUAUU \\
& F: UUCUCCGAACGUGUCACGUUT \\
GAPDH & R: ACGUGACACGUUCGGAGAATT \\
& F: GGAGCGAGATCCCTCCAAAAT \\
& R: GGCTGTTGTCATACTTCTCATGG
\end{tabular}

AKT1, AKT serine/threonine kinase 1; 3'UTR, 3' untranslated region; WT, wild-type; MUT, mutant; miR, microRNA; NC, negative control.

A

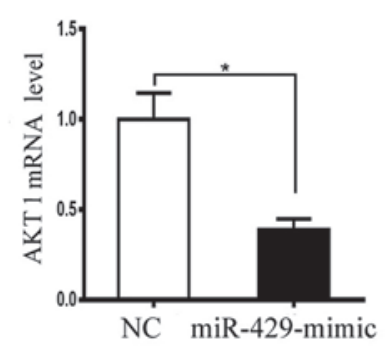

C

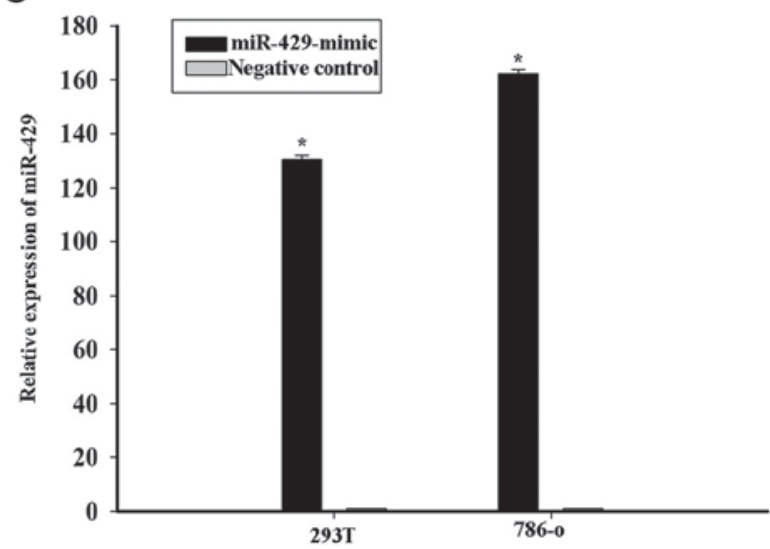

Figure 1. (A) 786-O cells transfected with miR-429 mimic exhibited decreased mRNA expression levels of AKT1 compared with those transfected with the negative control. (B) Overexpression of miR-429 resulted in a decrease in the protein expression levels of AKT1 in the renal cell carcinoma 786-O cell lines. (C) The fold changes to the expression levels of miR-429, as determined by quantitative PCR, were 130.4 and 162.3 in the $293 \mathrm{~T}$ and 786-O cells compared with the negative control group, respectively. The results represent three independent experiments. ${ }^{*} \mathrm{P} \leq 0.05$. miR, microRNA; AKT1, AKT serine/threonine kinase 1.

To perform luciferase reporter assay and validate the physical interaction of miR-429 with AKT1 mRNA, we constructed two vectors by cloning WT and MUT 3'-UTR into pGL3-promoter vector. Luciferase reporter constructs containing the 3'-UTR of AKT1 with the miR-429 mimic or negative control, either inhibitor or inhibitor NC, were transfected into the 293T cells. After $48 \mathrm{~h}$, the luciferase activity was detected. The relative luciferase activity of the reporter, containing the WT 3'-UTR was significantly suppressed when miR-429 was co-transfected into the $293 \mathrm{~T}$ cells compared with the negative control; containing the WT 3'-UTR was significantly promoted when inhibitor was cotransfected into the $293 \mathrm{~T}$ cells compared with inhibitor NC (Fig. 2B). By contrast, the luciferase activity of the MUT reporter was unaffected (Fig. 2C). All luciferase assays were repeated three times.

miR-429 suppressed 786-O cells derived xenograft tumor growth in vivo. A nude mouse xenograft model was established to confirm whether or not miR-429 overexpression could inhibit the growth of tumor. The results showed that miR-429 effectively inhibited the growth of tumor. The size and weight of nude mice with miR-429 treatment were lower than those of the control mice (Fig. 3A-C).

\section{Discussion}

RCC is a highly malignant digestive system tumor with radical surgical removal of the tumor as one of its main therapies. However, the recurrence rate of this disease remains high even after surgery. Therefore, searching for alternative treatment for $\mathrm{RCC}$ is urgent.

The role of miRNAs has been well studied in cancer and normal tissues (18-21). miRNAs are important biomarkers or therapeutic targets for RCC (22). However, the role of miR-429 is controversial. Machackova et al reported that miR-429 acts as a tumor suppressor by inhibiting the loss of E-cadherin in RCC cells (23). miR-429 can also inhibit the migration, invasion, and proliferation of RCC cells by targeting Sp1 directly (15). Furthermore, miR-429 is related to the resistance to chemoradiotherapy of pancreatic cancer through the 


\section{A C hsa-miR-429/AKT1 Alignment}

\begin{tabular}{|c|c|}
\hline $\begin{array}{l}\text { 3' ugccaaaauggucuGUCAUAAu 5' hsa-miR-429 } \\
\text { : IIIIII } \\
908: 5 \text { ' acaacauucaacuuUAGUAUUu } \\
\text { 3' AKT1 }\end{array}$ & $\begin{array}{ll}\text { mirSVR score: } & -0.1791 \\
\text { PhastCons score: } 0.6106\end{array}$ \\
\hline
\end{tabular}

B
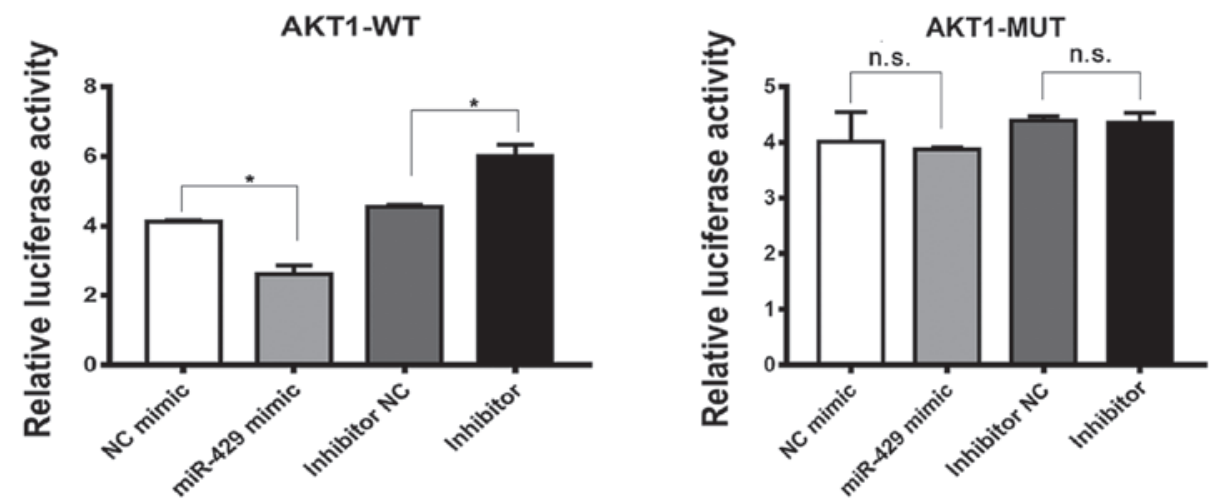

Figure 2. AKT1 is the target gene of miR-429. (A) Putative duplex formation between miR-429 and the AKT1 3'UTR. (B) Luciferase reporter constructs containing the 3'UTR of AKT1, together with the miR-429 mimic or negative control, inhibitor or inhibitor NC, were transfected into the 293T cells. After $48 \mathrm{~h}$, luciferase activity was detected. The relative luciferase activity of the reporter, containing the WT 3'UTR was significantly suppressed when miR-429 was cotransfected into the 293T cells compared with negative control; containing the WT 3'UTR was significantly promoted when inhibitor was cotransfected into the $293 \mathrm{~T}$ cells compared with inhibitor NC. (C) In contrast, the luciferase activity of the MUT reporter was unaffected. All luciferase assays were repeated three times; UTR, untranslated region; WT, wild-type; MUT, mutant. ${ }^{~} \mathrm{P}<0.05$. n.s., nonsignificant; AKT1, AKT serine/threonine kinase 1; miR, microRNA; UTR, untranslated region; NC, negative control; WT, wild-type; MUT, mutant.

$\mathbf{A}$

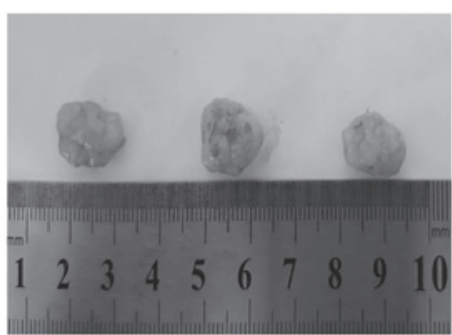

NC agomir

B

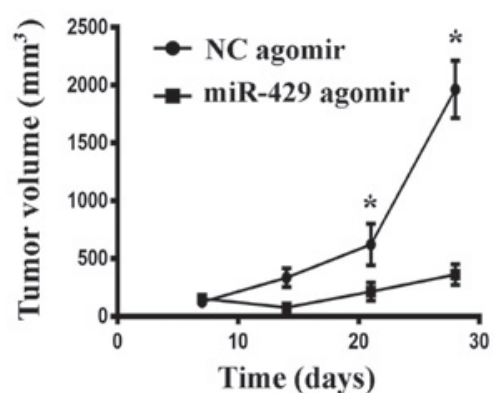

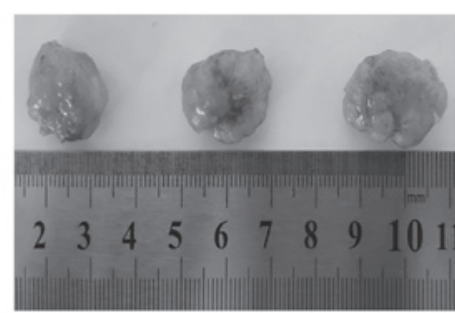

miR-429 agomir

C

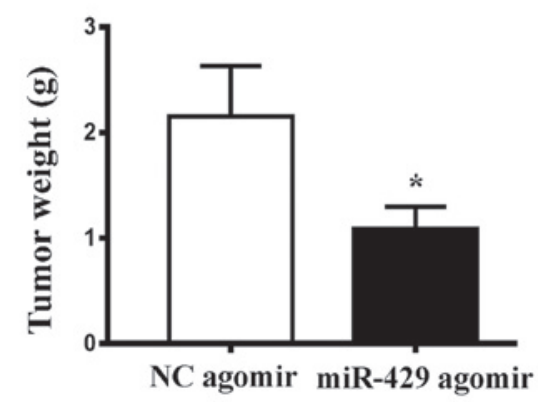

Figure 3. Tumor volumes at the (B) indicated dates and images and (A) tumor weight at (C) day 28 in the nude mice treated with intratumoral injection of miR-429 agomir or its negative control (negative control agomir) for a period of 4 weeks. The average values are present in the bar graphs (means \pm standard deviation; $\mathrm{n}=3$ for each group). The results represent three independent experiments. ${ }^{*} \mathrm{P} \leq 0.05$. miR, microRNA.

regulation of PDCD4 (24). In 2018, Xue and Tian reported that miR-429 expression is significantly lower in HCC tissues and cells than in normal tissues, and the elevated level of
miR-429 could inhibit metastasis in HCC cells by targeting RAB23 (14). By contrast, Tang et al reported that miR-429 enhances HCC cell invasion and migration by targeting the 
PTEN/PI3K/AKT/ $\beta$-catenin axis directly (25). In human prostate cancer cells, the elevated level of miR-429 promotes cell proliferation by targeting p27Kip1 (26).

In this study, we reported that miR-429 acts as a tumor suppressor in RCC cells. Several signal pathways, such as PDCD4 (24), VEGF (16), and c-myc (27), are involved in miR-429-regulated tumor development. This study first revealed that AKT1 is a target in mediating the anti-tumoral function of miR-429.

The phosphoinositide 3-kinase (PI3K)/RAC seine/ threonine-protein kinase (AKT)/mechanistic target of rapamycin (mTOR) signaling pathway is frequently dysregulated in several cancer types $(28,29)$. AKT plays a key role in the PI3K/AKT/mTOR signaling pathway and is closely associated with various cancer pathophysiologies, including EMT, growth, migration, metabolism, and angiogenesis (30-32). AKT has three isoforms (AKT1, AKT2, and AKT3) with different roles in cancer pathophysiology. AKT1 can also suppress breast cancer cell invasion and migration $(33,34)$ but accelerate the onset of tumorigenesis (35). Therefore, the knockdown of AKT1 gene inhibits the onset of tumorigenesis (36).

Four computational algorithms were used to explore the potential effect of miR-429 in renal tumorigenesis, predict the targets of miR-429, and confirm AKT1 as a novel target gene. The binding sites of miR-429 on the AKT1 3'-UTR were verified using the luciferase reporter assay. In addition, the cancer cells transfected with miR-429 mimics showed a reduced AKT1 expression at the mRNA and protein levels. The in vitro experiment showed that miR-429 overexpression could enhance the inhibition effect of tumor size and weight in nude mice. Hence, AKT1 is a direct target gene of miR-429 in renal caner. Therefore, miR-429/AKT1 axis can be a novel marker and therapeutic target in RCC treatment. However, owing to the experimental conditions were not perfect, there were no evaluated other proteins involved into the AKT pathway, such as Phospho-p70 S6 Kinase. When the conditions are improved, I will further explore components of the AKT pathway.

This study revealed that miR-429 acts as a tumor suppressor in RCC via the regulation of AKT1. This newly discovered underlying association between miR-429 and AKT1 may provide a new therapeutic strategy for RCC.

\section{Acknowledgements}

The authors would like to thank Miss Chen Gao (Department of Surgery, Peking University Shenzhen Hospital, Shenzhen, Guangdong, China) for technical assistance.

\section{Funding}

The current work was supported by funding from Science and Technology Plan Project of Guangzhou of China (grant no. 201510010177), Medical Science and Technology Program of Guangzhou of China (grant no. 20171A011092 and 20171A010325), Youth Scientific Research Project of Guangzhou Medical University of China (grant no. 2015A14) and the Medical Research Foundation of Guangdong Province of China (grant no. A2018093).

\section{Availability of data and materials}

All data generated or analyzed during the present study are included in this article.

\section{Authors' contributions}

ZS, GJ, XJ and GX conceived and designed the study. ZS and GX wrote the manuscript. JC, XL, HZ, ZF and YH performed the experiment. ZS, GJ, XJ and GX collected the clinical data. $\mathrm{ZS}$ and GX reviewed and edited the manuscript. All authors read and approved the manuscript.

\section{Ethics approval and consent to participate}

The study protocol conformed to the guidelines of the Guangzhou Medical University of China Ethics Committee, and it was approved by the review board of Guangzhou Medical University of China (approval no. 2017-020). Written informed consent was obtained from all patients.

\section{Patient consent for publication}

Written informed consent was obtained from all patients for the publication of their data.

\section{Competing interests}

The authors declare that they have no competing interests.

\section{References}

1. Gupta K, Miller JD, Li JZ, Russell MW and Charbonneau C: Epidemiologic and socioeconomic burden of metastatic renal cell carcinoma (mRCC): A literature review. Cancer Treat Rev 34: 193-205, 2008.

2. Wei C, Wu S, Li X, Wang Y, Ren R, Lai Y and Ye J: High expression of FER tyrosine kinase predicts poor prognosis in clear cell renal cell carcinoma. Oncol Lett 5: 473-478, 2013.

3. Bouyssou JM, Manier S, Huynh D, Issa S, Roccaro AM and Ghobrial IM: Regulation of microRNAs in cancer metastasis. Biochim Biophys Acta 1845: 255-265, 2014.

4. Schmidt U and Begley CG: Cancer diagnosis and microarrays. Int J Biochem Cell Biol 35: 119-124, 2003.

5. Yu X, Zhang X, Bi T, Ding Y, Zhao J, Wang C, Jia T, Han D, Guo G, Wang B, et al: MiRNA expression signature for potentially predicting the prognosis of ovarian serous carcinoma. Tumour Biol 34: 3501-3508, 2013.

6. Walter BA, Valera VA, Pinto PA and Merino MJ: Comprehensive microRNA profiling of prostate cancer. J Cancer 4: 350-357, 2013.

7. Miao J, Wu S, Peng Z, Tania M and Zhang C: MicroRNAs in osteosarcoma: Diagnostic and therapeutic aspects. Tumour Biol 34: 2093-2098, 2013.

8. $\mathrm{Li} \mathrm{L}$ and Li W: Epithelial-mesenchymal transition in human cancer: Comprehensive reprogramming of metabolism, epigenetics, and differentiation. Pharmacol Ther 150: 33-46, 2015.

9. Chen H, Xia B, Liu T, Lin M and Lou G: KIAA0101, a target gene of miR-429, enhances migration and chemoresistance of epithelial ovarian cancer cells. Cancer Cell Int 16: 74, 2016.

10. Fan JY, Fan YJ, Wang XL, Xie H, Gao HJ, Zhang Y, Liu M and Tang H: miR-429 is involved in regulation of NF- $\kappa$ B activity by targeting IKK $\beta$ and suppresses oncogenic activity in cervical cancer cells. FEBS Lett 591: 118-128, 2017.

11. Goeppert B, Ernst C, Baer C, Roessler S, Renner M, Mehrabi A, Hafezi M, Pathil A, Warth A, Stenzinger A, et al: Cadherin-6 is a putative tumor suppressor and target of epigenetically dysregulated miR-429 in cholangiocarcinoma. Epigenetics 11: 780-790, 2016. 
12. Xiao P, Liu W and Zhou H: miR-200b inhibits migration and invasion in non-small cell lung cancer cells via targeting FSCN1. Mol Med Rep 14: 1835-1840, 2016.

13. Sheng N, Zhang L and Yang S: MicroRNA-429 decreases the invasion ability of gastric cancer cell line BGC-823 by downregulating the expression of heparanase. Exp Ther Med 15: 1927-1933, 2018.

14. Xue $\mathrm{H}$ and Tian GY: MiR-429 regulates the metastasis and EMT of HCC cells through targeting RAB23. Arch Biochem Biophys 637: 48-55, 2018

15. Wu D, Niu X, Pan H, Zhou Y, Zhang Z, Qu P and Zhou J: Tumor-suppressing effects of microRNA-429 in human renal cell carcinoma via the downregulation of Sp1. Oncol Lett 12 : 2906-2911, 2016

16. Chen D, Li Y, Li Y, Jin L, Su Z, Yu Z, Yang S, Mao X and Lai Y: Tumor suppressive microRNA-429 regulates cellular function by targeting VEGF in clear cell renal cell carcinoma. Mol Med Rep 13: 1361-1366, 2016.

17. Livak KJ and Schmittgen TD: Analysis of relative gene expression data using real-time quantitative PCR and the 2(-Delta Delta c(T)) method. Methods 25: 402-408, 2001.

18. Liu K, Liu S, Zhang W, Jia B, Tan L, Jin Z and Liu Y: miR-494 promotes cell proliferation, migration and invasion, and increased sorafenib resistance in hepatocellular carcinoma by targeting PTEN. Oncol Rep 34: 1003-1010, 2015.

19. Meng W, Tai Y, Zhao H, Fu B, Zhang T, Liu W, Li H, Yang Y, Zhang Q, Feng Y, et al: Downregulation of miR-33a-5p in hepatocellular carcinoma: A possible mechanism for chemotherapy resistance. Med Sci Monit 23: 1295-1304, 2017.

20. Qin CZ, Lou XY, Lv QL, Cheng L, Wu NY, Hu L and Zhou HH: MicroRNA-184 acts as a potential diagnostic and prognostic marker in epithelial ovarian cancer and regulates cell proliferation, apoptosis and inflammation. Pharmazie 70 668-673, 2015.

21. Guo Y, Pang Y, Gao X, Zhao M, Zhang X, Zhang H, Xuan B and Wang Y: MicroRNA-137 chemosensitizes colon cancer cells to the chemotherapeutic drug oxaliplatin (OXA) by targeting YBX1. Cancer Biomark 18: 1-9, 2017.

22. Chan SH and Wang LH: Regulation of cancer metastasis by microRNAs. J Biomed Sci 22: 9, 2015.

23. Machackova T, Mlcochova H, Stanik M, Dolezel J, Fedorko M, Pacik D, Poprach A, Svoboda M and Slaby O: MiR-429 is linked to metastasis and poor prognosis in renal cell carcinoma by affecting epithelial-mesenchymal transition. Tumour Biol 37: 14653-14658, 2016.
24. Yu G, Jia B, Cheng Y, Zhou L, Qian B, Liu Z and Wang Y: MicroRNA-429 sensitizes pancreatic cancer cells to gemcitabine through regulation of PDCD4. Am J Transl Res 9: 5048-5055, 2017.

25. Tang J, Li L, Huang W, Sui C, Yang Y, Lin X, Hou G, Chen X, Fu J, Yuan S, et al: MiR-429 increases the metastatic capability of HCC via regulating classic Wnt pathway rather than epithelial-mesenchymal transition. Cancer Lett 364: 33-43, 2015.

26. Ouyang Y, Gao P, Zhu B, Chen X, Lin F, Wang X, Wei J and Zhang H: Downregulation of microRNA-429 inhibits cell proliferation by targeting p27Kip1 in human prostate cancer cells. Mol Med Rep 11: 1435-1441, 2015.

27. Sun T, Wang C, Xing J and Wu D: miR-429 modulates the expression of c-myc in human gastric carcinoma cells. Eur J Cancer 47: 2552-2559, 2011

28. Bhaskar PT and Hay N: The two TORCs and Akt. Dev Cell 12: 487-502, 2007.

29. Engelman JA: Targeting PI3K signalling in cancer: Opportunities, challenges and limitations. Nat Rev Cancer 9: 550-562, 2009.

30. Tang H, Massi D, Hemmings BA, Mandalà M, Hu Z, Wicki A and Xue G: AKT-ions with a TWIST between EMT and MET. Oncotarget 7: 62767-62777, 2016.

31. Xu N, Lao Y, Zhang Y and Gillespie DA: Akt: A double-edged sword in cell proliferation and genome stability. J Oncol 2012: 951724, 2012.

32. Manning BD and Cantley LC: AKT/PKB signaling: Navigating downstream. Cell 129: 1261-1274, 2007.

33. Chin YR and Toker A: The actin-bundling protein palladin is an Akt1-specific substrate that regulates breast cancer cell migration. Mol Cell 38: 333-344, 2010.

34. Liu H, Radisky DC, Nelson CM, Zhang H, Fata JE, Roth RA and Bissell MJ: Mechanism of Aktl inhibition of breast cancer cell invasion reveals a protumorigenic role for TSC2. Proc Natl Acad Sci USA 103: 4134-4139, 2006

35. Hutchinson JN, Jin J, Cardiff RD, Woodgett JR and Muller WJ: Activation of Akt-1 (PKB-alpha) can accelerate ErbB-2-mediated mammary tumorigenesis but suppresses tumor invasion. Cancer Res 64: 3171-3178, 2004.

36. Maroulakou IG, Oemler W, Naber SP and Tsichlis PN: Akt1 ablation inhibits, whereas Akt2 ablation accelerates, the development of mammary adenocarcinomas in mouse mammary tumor virus (MMTV)-ErbB2/neu and MMTV-polyoma middle $\mathrm{T}$ transgenic mice. Cancer Res 67: 167-177, 2007. 